\title{
Residential outdoor education and environmental attitudes: an examination in a Malaysian University
}

\begin{abstract}
While many outdoor educators are confident their residential outdoor education (ROE) programs improve participants' environmental attitudes, research findings are inconclusive. This study seeks to examine the effects of a Malaysian university ROE camp on environmental attitudes. A pre and post test approach with non equivalent control group design was utilized among 176 first year undergraduate students. A modified version of CHEAKS and open ended questionnaire were used to gather data from pre and post-tests. Results from ANCOVA procedures suggested that the camp had positively improved the experimental group's environmental attitudes. Issues of bridging ROE learning outcomes into daily practices are addressed, and a framework for future practice and research is suggested.
\end{abstract}

Keyword: Environmental attitudes; Residential outdoor education; University students. 